Chapter 10

\title{
The Extracellular Indolic Compounds of Lentinus edodes
}

\author{
Olga M. Tsivileva, Ekaterina A. Loshchinina and \\ Valentina E. Nikitina \\ Additional information is available at the end of the chapter \\ http://dx.doi.org/10.5772/53262
}

\section{Introduction}

In macrobasidial fungi, the properties of compounds of a phytohormonal nature, which are well known in higher plants and are intensively studied in soil associative microorganisms, are only described in an unsystematic manner and, apparently, to an insufficient degree. Auxins are the most studied group of phytohormonal substances. As an object of research, along with other mycological objects of industrial cultivation, the higher fungus-xylotrophic basidiomycete Lentinus edodes (Berk.) Sing (Lentinula edodes (Berk.) Pegler or shiitake), which is of high practical importance and the physiological and biochemical characteristics of which are obviously insufficiently studied, is of particular interest.

For long enough, there have been speculations that phytohormones, including representatives of the auxin group, are involved in the processes of cell growth and cytodifferentiation not only in plants, but also in fungi. Nevertheless, this issue still remains practically unstudied.

Of particular interest are the effects and mechanisms of the action of biologically active substances at low doses. At small and ultrasmall concentrations $\left(10^{-20}-10^{-13} \mathrm{M}\right)$, there is a manifestation of the activity of many natural chemomediators - toxins and antidotes, substances warning of danger, pheromones, cryoprotectants, and other compounds, including phytohormones [1]. There is a description of the paradoxical nature of the effect of low concentrations of toxic substances and drugs, which is particularly expressed in the bimodal or polymodal dependence dose-effect. It is noted in [2] that the consequences of the effects of small doses of xenobiotics may be no less serious than the consequences of high single doses: under their influence, essential links may change and some adaptation systems may fail, because the body is only able to adapt to effects, which are in the usual range of action. 
There are two main ways of biosynthesis of phytohormone of indole-3-acetic acid (IAA), namely tryptophan-dependent (Trp-dependent), in the case of which amino acid tryptophan serves as a precursor to IAA, and tryptophan-independent (Trp-independent), in the case of which IAA is produced from indole, anthranilic acid, and indole-3-glycerophosphate [3]. Trp-dependent synthesis of IAA by microorganisms can take place in one of the following four ways (see the scheme below): by indole-3-pyruvic acid and indole-3-acetaldehyde (the most common way), by tryptamine and indole-3-acetaldehyde, by indole-3-acetamide, and by indole-3-acetonitrile. According to some reports, indolylacetaldoxime may also be converted into IAA through indole acetaldehyde $[4,5]$.

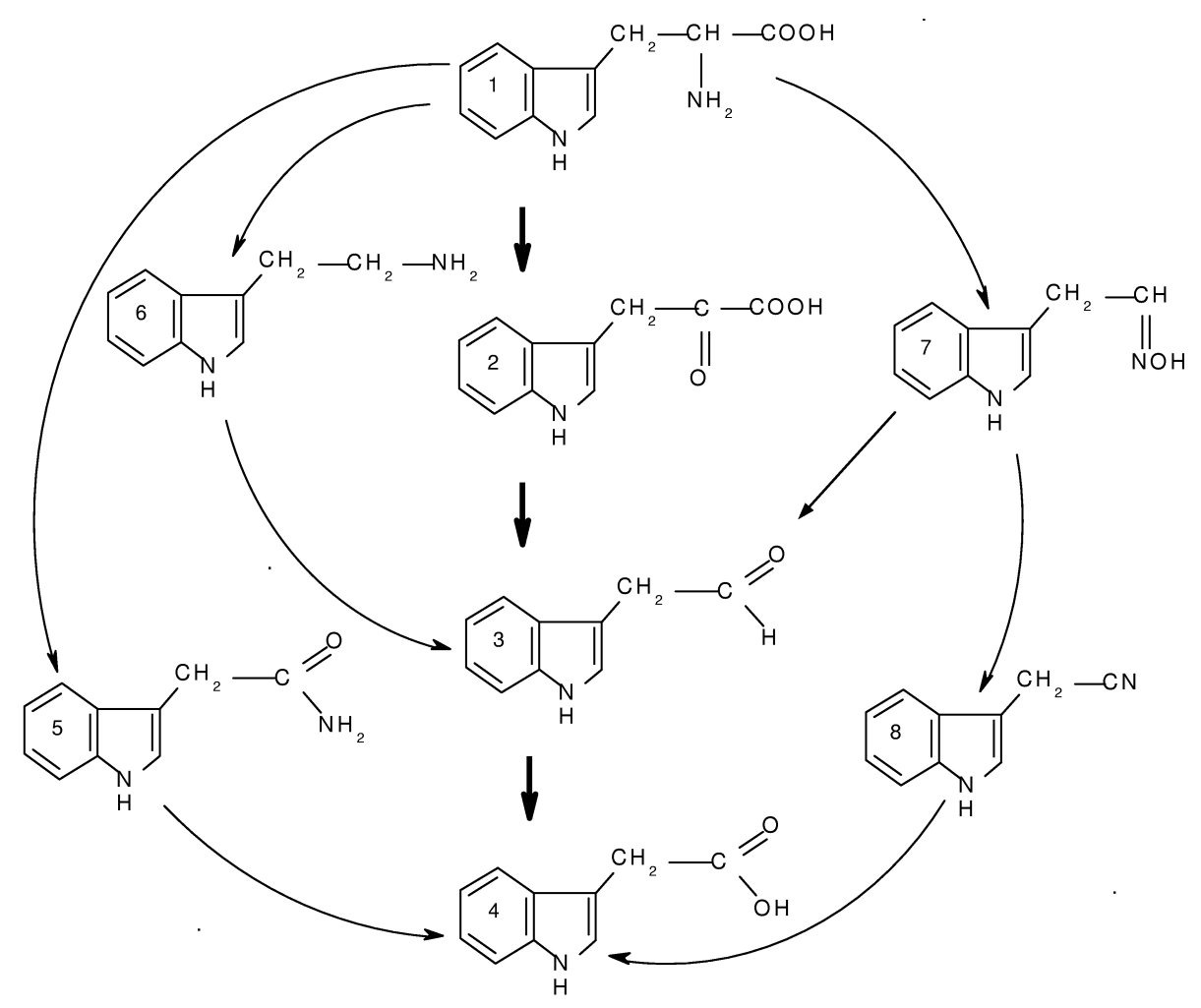

\footnotetext{
$\longrightarrow$ principal pathway of IAA biosynthesis

$\longrightarrow$ additional pathways
}

Scheme 1. Tryptophan-dependent biosynthesis of indolylacetic acid in microorganisms. 1 - tryptophan, 2 -indolylpyruvic acid, 3 - indolylacetaldehyde, 4 - indolylacetic acid, 5 -indolylacetamide, 6 - tryptamine, 7 -indolylacetaldoxime, 8 - indolylacetonitrile 
Many phytopathogenic fungi and bacteria have multiple ways of IAA biosynthesis. The ways of IAA biosynthesis in macrobasidiomycetes have been at best considered only at the level of qualitative effect of tryptophan supplementation to a growth medium.

The purpose of the work is to study the composition of a group of indole metabolites, which accompany the production of IAA by the basidiomycete Lentinus edodes, and to establish whether this way of biosynthesis of IAA is Trp-dependent or there is a switching to the Trpindependent way during growing xylotroph in the presence of exogenous synthetic analogs of compounds - precursors to IAA.

\section{Experimental}

A culture of Lentinus edodes (strain F-249) obtained from the collection of macrobasidiomycetes of the Department of Mycology and Algology, Moscow State University (Russia) was used. This fungal culture was maintained on wort agar at $4^{\circ} \mathrm{C}$.

As an inoculum, a 14-day culture of L. edodes grown on beer-wort agar $\left(4^{\circ} \mathrm{Bx}\right)$ was used. The temperature of growing was $26^{\circ} \mathrm{C}$. The agar blocks with mycelium were cut out in sterile conditions using a metal punch with a diameter of $5 \mathrm{~mm}$, and served for inoculation of liquid nutritive media at a rate of two blocks for $20 \mathrm{ml}$ of medium.

The submerged culture of the fungus was grown in synthetic medium $(9 \mathrm{~g} / \mathrm{l}$ of glucose and $1.5 \mathrm{~g} / \mathrm{l}$ of L-asparagine), as well as in beer-wort $\left(1.2^{\circ} \mathrm{Bx}\right)$. To determine the dry biomass, the mycelium was filtered, weighed on an analytical balance, and dried to a constant weight. To study the effect of compounds of indolic nature, we added them as solutions in ethanol- $\mathrm{H}_{2} \mathrm{O}$ $(1: 1, v / v)$ mixtures to the medium subjected to autoclave glucose and asparagine immediately before planting in sterile conditions. The concentrations of indolic compounds in the culture medium were $0.1,1,10$, and $100 \mathrm{mg} / \mathrm{l}$. The effect of IAA on the growth of the culture was studied in the range of $10^{-8}-10^{-1} \mathrm{~g} / \mathrm{l}$.

The indolic compounds were determined in the culture fluid by high performance liquid chromatography (HPLC) using pure commercial preparations of IAA, Trp, tryptamine (TAM), indolylacetamide (IAAm), indolylpyruvic acid (IPyA), indolylacetaldehyde (IAAld), indole, indole-3-acetonitrile, anthranilic acid, and 5-hydroxy-indole-3-acetic acid (5-hydroxy-IAA) as standards. For the identification and quantification of indolic compounds, samples of the culture fluid were collected under sterile conditions during its growth; then, they were filtered by membrane filters type $0.22 \mu \mathrm{M}$ GVPP (Millipore, Ireland) and analyzed. Junction reverse-phase HPLC was performed on a medium with chemically bound hydrophobic residues of C18 $(5 \mu \mathrm{M})$. The column $(150 \times 4.6 \mathrm{~mm})$ Luna $5 \mu$ C18 (2) (Phenomenex, United States) equipped with a precolumn (type of "security guard") of the same brand. As an eluent, a mixture of methanol and water was used (36:64 or $50: 50, v / v)$. A UV absorbance detector operating in the wavelength range of 250-300 $\mathrm{nm}$ was used. The sample volume was $20 \mu \mathrm{l}$, and the pressure was $12 \mathrm{MPa}$. 
Mass $(m / V)$ expression of concentration of indolic compounds in liquid media was used. The selected method of expressing concentrations is used in most published articles relevant to the subject presented in this work and, therefore, allows for comparisons in the most convenient form [3-5].

\section{Results and discussion}

\subsection{Intermediate products of biosynthesis of IAA in L. edodes}

Our assumptions about the existence of Trp-dependent synthesis of IAA in the fungus were based on the following observations:

1. A phenomenon of biosynthesis of extracellular Trp by the studied strain of L. edodes was discovered. In a synthetic medium, initially not containing this amino acid, the concentrations of Trp ranged from $14 \mathrm{mg} / \mathrm{l}$ on the 7 th day to $24 \mathrm{mg} / \mathrm{l}$ on the 21st day. A reduction in the concentration was observed on the 7th and 14th days. For all studied ages of the culture, the introduction of Trp additives (10 and $100 \mathrm{mg} / \mathrm{l})$ into the medium resulted in a significant increase in the content of this substance in the culture fluid compared to the initial one. The maximum quantity of Trp (about $330 \mathrm{mg} / \mathrm{l}$ ) was observed on the 14th day in the medium supplemented with $100 \mathrm{mg} / \mathrm{l}$ of this acid.

2. It was revealed that the submerged culture of L. edodes F-249, growing on a glucose and asparagine medium, was capable of forming extracellular IAA. In a control experiment, the highest concentration of auxin (about $7.5 \mathrm{mg} / \mathrm{l}$ ) was observed on the 21st day. At an exogenous introduction of Trp into the culture medium, the content of IAA increased and a maximum $(9.4 \mathrm{mg} / \mathrm{l})$ was reached on the 14 th day in the medium supplemented with $100 \mathrm{mg} / \mathrm{l}$ of amino acid, i.e., with the greatest concentration of Trp.

Thus, at the moment when the method for phytohormone formation by the fungus culture started to become clear, we discovered the biosynthetic ability of the fungus with regard to IAA and its precursor Trp.

The main known methods of IAA biosynthesis are associated with Trp. A method, which is independent of tryptophan (Trp-independent), occurs in plants and among the bacteria detected in azospirilla and cyanobacteria. To date, the prevailing view is that the contribution of the Trp-independent way to IAA biosynthesis is not significant; the mechanism of auxin biosynthesis has not been studied. Nevertheless, researchers' opinions are divided. Thus, although previous works prove the existence of an indolylacetamide way in Azospirillum brasilense [6]; give biochemical and genetic grounds for the usage of the way via IPyA by azospirilla [7, 8]; and, at the same time, make an assumption that $90 \%$ of the IAA in Azospirillum is biosynthesized in the tryptophan-independent way [6].

The data we obtained earlier [9] showed that in the L. edodes F-249 culture medium, there were intermediate formations as a result of three ways of Trp-dependent biosynthesis of IAA - through TAM, IAAm, and IPyA. This is consistent with the known data, according to 
which the ability to synthesize IAA simultaneously in several different reactions is found in various microorganisms, including some fungi. Interestingly, indolylacetaldehyde, which is an intermediate in the IAA synthesis from both IPyA and TAM, is only found in media supplemented with IPyA, where IAAld is accumulated in large quantities, but the level of IAA is at the same time very low. The way via IPyA is not completely realized. None of the samples studied had indole-3-acetonitrile, which is another intermediate of IAA synthesis from Trp. This is consistent with the data of literature, according to which cases of IAA synthesis by this intermediate have not yet been identified in fungi [5].

\subsection{Prerequisites of the Trp-independent way of IAA synthesis in Shiitake}

Our assumptions about the possibility of the existence of an IAA biosynthesis way distinct from the Trp-dependent one in the studied fungal culture were initially based on the following: It is considered in $[10,11]$ that the bacterial synthesis of IAA is a way to detoxify tryptophan. According to some authors [12], in bacteria, such as A. brasilense, there is no way of Trp degradation, which is toxic to them, but it can be transformed into IAA. Therefore, for bacterial producers, Trp is the most effective and "rational" precursor to IAA [13].

For the higher fungi under our study, in contrast to azospirilla, Trp is not toxic at least up to relatively high concentrations, which a fungal culture creates during submerged cultivation, i.e., up to $330 \mu \mathrm{g} / \mathrm{ml}$ (see above).

The Trp-independent way is connected to the synthesis of IAA from indole, anthranilic acid, and indole-3-glycerophosphate. One of the hardest things in proving the Trp-independence of IAA biosynthesis is that indole is a substance, which can serve as both a precursor to IAA in Trp-independent biosynthesis and a precursor to tryptophan. Then, Trp synthesized from indole can also serve as a precursor for IAA [14].

As a result of the study of IAA biosynthesis by bacteria, many authors have come to a conclusion that allows them to make judgments concerning the preferred substances-precursors of IAA in the context of its biosynthesis. The arguments are as follows:

1. Bacteria cannot produce IAA by the Trp-independent way when cultured with indole as a precursor to this phytohormone, because IAA synthesis is not stimulated while growing bacteria in media containing indole [13]. This suggests that indole is not a preferred precursor to IAA compared to Trp.

2. The use of anthranilic acid or indole for the synthesis of IAA by the non-tryptophan way using microorganisms is unlikely, because the presence of Trp in the culture fluid is demonstrated in all experiments.

On the basis of the experimental data obtained in the present paper, one can provide arguments for and against the coexistence of two alternative ways of IAA biosynthesis in L. edodes, Trp-dependent and Trp-independent. The media with the addition of indole were characterized by fairly high values of IAA (up to $9 \mathrm{mg} / \mathrm{l}$ ) on the 14th - 21st days of cultivation, and the amounts of formed IAA were not dependent on the initial concentration of indole. The medium with $100 \mathrm{mg} / \mathrm{l}$ of indole was an exception, where the level of IAA 
decreased on the 21st day and the concentration of IPyA at this point increased dramatically. The data are presented in the Table.

\begin{tabular}{|c|c|c|c|c|c|c|}
\hline Experimental & & Final cor & ntration in th & e culture fluid & $\mathrm{l}, \mathrm{mg} / \mathrm{l}$ & \\
\hline $\begin{array}{l}\text { Indolic additive, } \\
\text { mg/l }\end{array}$ & $\begin{array}{c}\text { Cultivation time, } \\
\text { days }\end{array}$ & Tryptamine & $\begin{array}{l}\text { Indolyl- } \\
\text { acetamide }\end{array}$ & $\begin{array}{c}\text { Indolyl-acetic } \\
\text { acid }\end{array}$ & $\begin{array}{l}\text { Indolyl- } \\
\text { pyruvic acid }\end{array}$ & Tryptophan \\
\hline \multirow{5}{*}{$\begin{array}{l}\text { Control (medium } \\
\text { without indole) }\end{array}$} & 3 & 3.7 & 3.3 & 3.7 & $\mathrm{n} / \mathrm{d}$ & 19.5 \\
\hline & 7 & 3.8 & 3.2 & $n / d$ & $\mathrm{n} / \mathrm{d}$ & 13.8 \\
\hline & 10 & 4.2 & 3.1 & $\mathrm{n} / \mathrm{d}$ & $\mathrm{n} / \mathrm{d}$ & 18.6 \\
\hline & 14 & 3.4 & 2.3 & 3.7 & 0.9 & 16.9 \\
\hline & 21 & 3.9 & 3.7 & 7.4 & 7.7 & 23.9 \\
\hline \multirow{5}{*}{0.1} & 3 & 3.1 & 3.4 & 4.0 & 1.0 & 17.5 \\
\hline & 7 & 1.3 & $\mathrm{n} / \mathrm{d}$ & $n / d$ & 0.3 & 9.5 \\
\hline & 10 & 1.9 & $\mathrm{n} / \mathrm{d}$ & $n / d$ & 0.7 & 10.8 \\
\hline & 14 & 2.5 & 2.6 & 6.9 & 5.3 & 16.1 \\
\hline & 21 & 3.3 & 3.4 & 9.0 & 11.6 & 20.5 \\
\hline \multirow{5}{*}{1} & 3 & 2.5 & 2.7 & 5.5 & $n / d$ & 14.8 \\
\hline & 7 & 1,3 & $\mathrm{n} / \mathrm{d}$ & $n / d$ & 0.4 & 8.3 \\
\hline & 10 & 1.5 & $\mathrm{n} / \mathrm{d}$ & $n / d$ & 0.9 & 9.8 \\
\hline & 14 & 2.3 & 2.3 & 5.6 & 2.8 & 16.2 \\
\hline & 21 & 2.9 & 2.7 & 7.4 & 5.9 & 16.7 \\
\hline \multirow{5}{*}{10} & 3 & 2.9 & 3.0 & 3.4 & 0.5 & 17.1 \\
\hline & 7 & 1.9 & $\mathrm{n} / \mathrm{d}$ & $n / d$ & 0.3 & 9.9 \\
\hline & 10 & 1.8 & $\mathrm{n} / \mathrm{d}$ & $n / d$ & 0.9 & 7.9 \\
\hline & 14 & 2.4 & 2.2 & 6.2 & 4.7 & 15.6 \\
\hline & 21 & 3.7 & 2.7 & 8.1 & 7.7 & 17.2 \\
\hline \multirow{5}{*}{100} & 3 & 3.1 & 3.5 & $n / d$ & 0.2 & 13.9 \\
\hline & 7 & 1.2 & $n / d$ & $n / d$ & 0.4 & 13.9 \\
\hline & 10 & 1.2 & $\mathrm{n} / \mathrm{d}$ & $n / d$ & 0.4 & 10.1 \\
\hline & 14 & 2.4 & 2.2 & 6.1 & 4.2 & 15.5 \\
\hline & 21 & 3.3 & 2.2 & 2.2 & 16.7 & 19.1 \\
\hline
\end{tabular}

Note: $\mathrm{n} / \mathrm{d}$ - not detectable

Table 1. Effect of indole on the formation of extracellular indole compounds by the mushroom Lentinus edodes F-249 
Thus, the indole stimulated the synthesis of IAA. In our experiments on a synthetic medium, initially not containing this substance, the concentrations of extracellular Trp ranged from $13.8 \mathrm{mg} / \mathrm{l}$ on the 7 th day to $9.23 \mathrm{mg} / \mathrm{l}$ on the 21 st day. This means that there were no formally noted cases of IAA synthesis in the absence of tryptophan and the Trp-dependent way of IAA biosynthesis took place in the studied fungal culture. Nevertheless, one can assume that there is a transfer to the Trp-independent way or a connection between this alternative ways (which is likely), realizing in the presence of exogenous indole within the concentration range of $1 \bullet 10^{-3}-1 \bullet 10^{-4} \mathrm{~g} / \mathrm{l}$ (Table).

It is necessary to note the following about the additions of indole: in this case, a background level of Trp also exists, but, firstly, it is virtually unchanged compared to the control experiment and the IAA synthesis is by a factor of 1.5-1.9 greater (Fig. 1a).
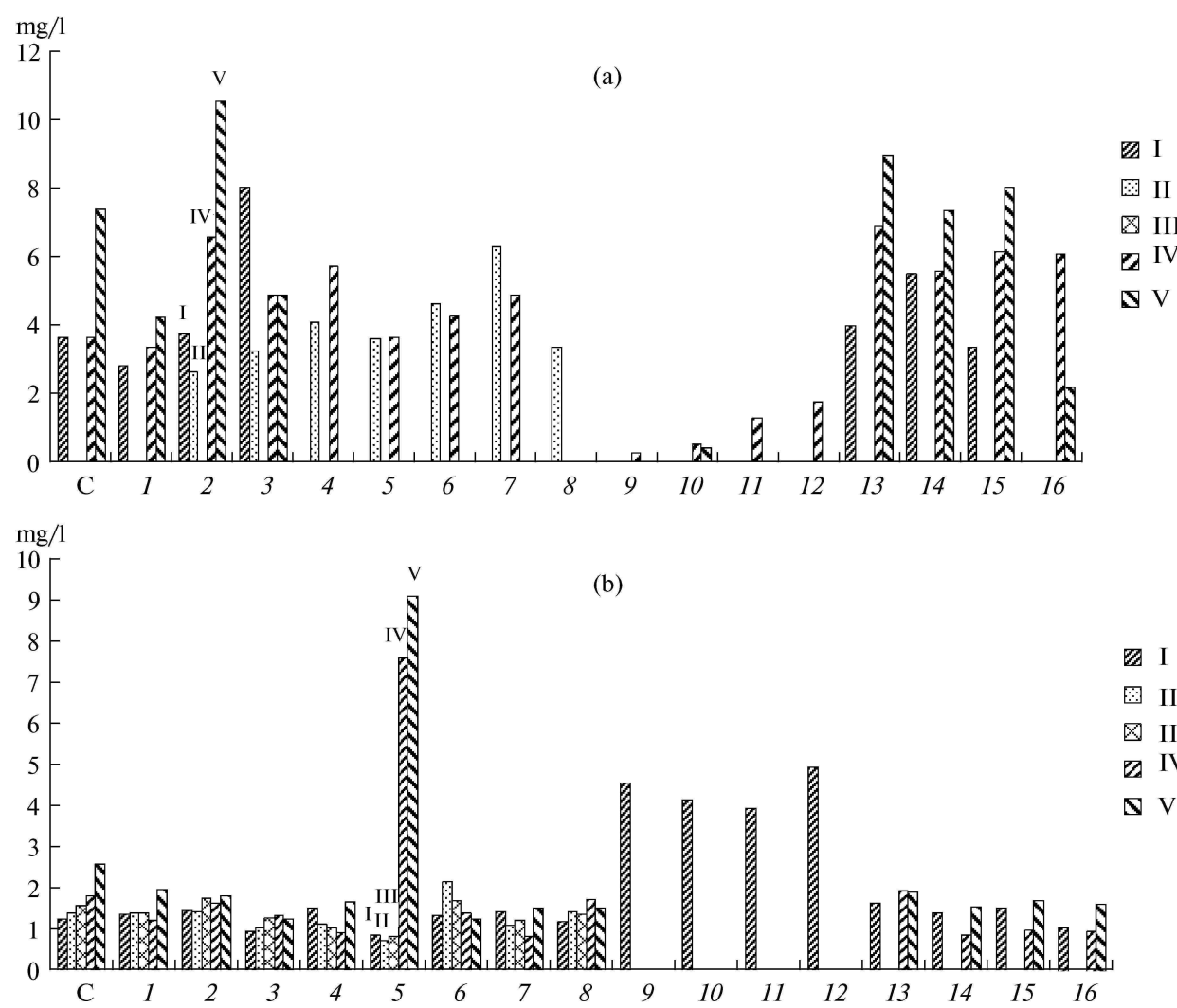

Figure 1. Synthesis (mg/l) of (a) extracellular indolylacetic acid, and (b) 5-hydroxy-indolylacetic acid by submerged cultures of Lentinus edodes F-249 on media with an addition of indolic compounds at different durations of cultivation (days): (I) 3, (II) 7, (III) 10, (IV) 14, and (V) 21. C - control; tryptamine of (1) 0.1, (2) 1, (3) 10, and (4) 100 mg/l; indolylacetamide of (5) $0.1,(6) 1,(7) 10$, and (8) $100 \mathrm{mg} / \mathrm{l}$; indolylpyruvic acid of (9) 0.1, (10) 1, (11) 10, and (12) 1000 mg/l; indole of (13) $0.1,(14) 1,(15) 10$, and (16) $100 \mathrm{mg} / \mathrm{l}$. 
This happens in spite of the reduced fungal biomass under the influence of indole, which clearly had no positive effect on the growth rates of L. edodes. It caused a significant decrease in the biomass compared to the control (up to $34 \%$ in the medium with $100 \mathrm{mg} / \mathrm{l}$ of indole) and strongly inhibited the growth of the culture (Fig. 2).

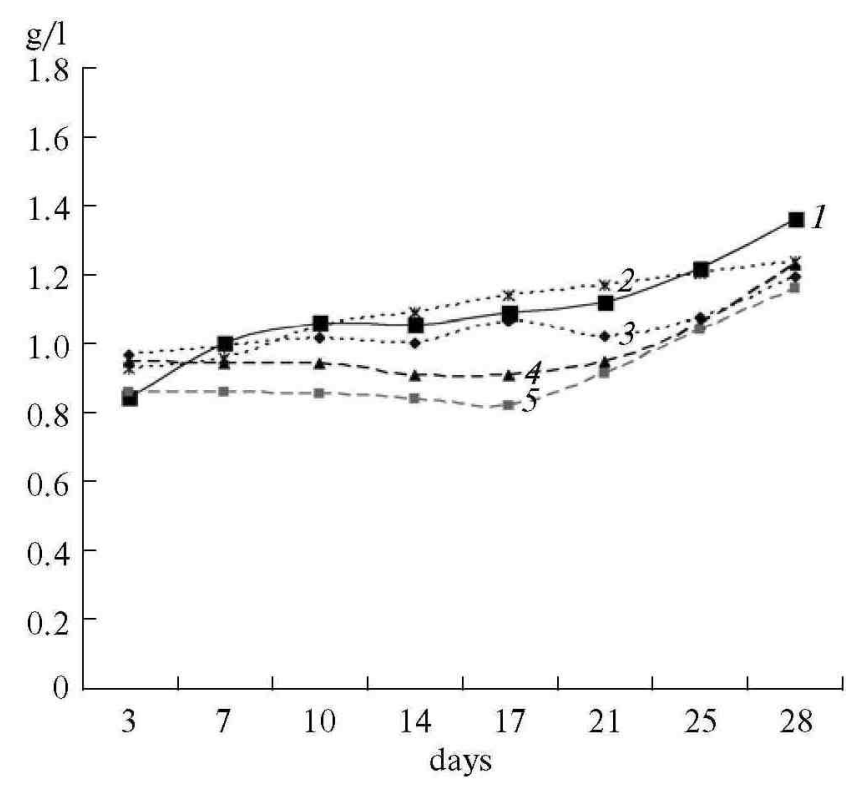

Figure 2. Mycelial biomass accumulation ( $\mathrm{g} / \mathrm{l})$ by submerged cultures of Lentinus edodes F-249 of different ages (days) on media with an indole addition (mg/L): (1) 0, (2) 0.1, (3)1, (4) 10, and (5) 100.

Secondly, the concentration of $\operatorname{Trp}$ in the medium completely remains the same when the level of exogenous indole is increased by 1000 times (from 0.1 to $100 \mathrm{mg} / \mathrm{l}$ ), and, therefore, indole is not presumably a significant precursor to Trp in this case (with further transformation of the latter into IAA). The elevated level of indole in the culture medium was increasing the biosynthesis of IAA, but not tryptophan. Thus, we are talking about the connection of the Trp-independent way.

Not only in the case of exogenous indole there are signs of this way, but also while inducing IAA biosynthesis with its exogenous microadditives $\left(1 \bullet 10^{-5}-1 \bullet 10^{-8} \mathrm{~g} / \mathrm{l}\right)$. For example, at an initial IAA concentration of $1 \bullet 10^{-7} \mathrm{~g} / \mathrm{l}$ on the tenth day of growth, the level of phytohormone was about $4 \bullet 10^{-4} \mathrm{~g} / \mathrm{l}$; i.e., it had increased by a factor of 4000 (Fig. 3a).

The appearance of anthranilic acid (up to $1.5 \mathrm{mg} / \mathrm{l}$ ) as a sign of the Trp-independent path was only marked by us under these experimental conditions (Fig. 3b). At the same time, Trp itself was not detected in the culture fluid in any of the eight tested concentrations of IAA additive. 


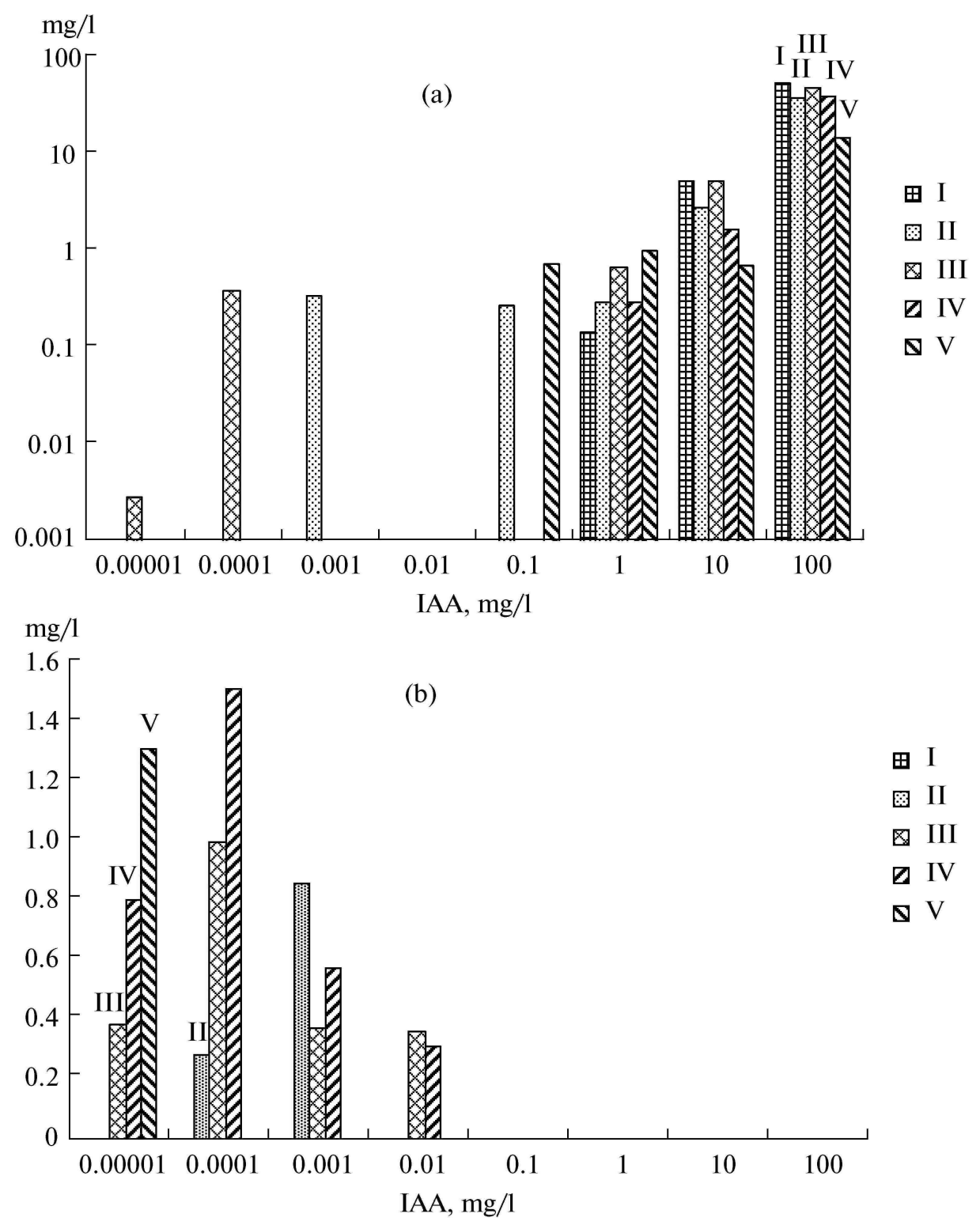

Figure 3. Effect of exogenous indolylacetic acid additives ( $\mathrm{mg} / \mathrm{l})$ on the content ( $\mathrm{mg} / \mathrm{l})$ of (a) indolylacetic acid, and (b) anthranilic acid in the cultural fluid of Lentinus edodes F-249 of different ages (days): (I) 3, (II) 7, (III) 10, (IV) 14, and (V) 21.

Besides anthranilic acid, IPyA is also synthesized in the presence of low concentrations of IAA $\left(1 \bullet 10^{-4}-1 \bullet 10^{-8} \mathrm{~g} / \mathrm{l}\right)$, but the appearance of IAAld - a product of the conversion of IPyA during Trp-dependent IAA synthesis - was not observed. This situation with IAAld changes 
at the higher concentrations $\left(1 \bullet 10^{-4}-1 \bullet 10^{-1} \mathrm{~g} / \mathrm{l}\right)$ of exogenous IAA, when on the tenth day of growth, for example, there accumulated from 3.6 to $8.7 \mathrm{mg} / \mathrm{l}$ of IAAld (Fig. $4 \mathrm{a}$ ).

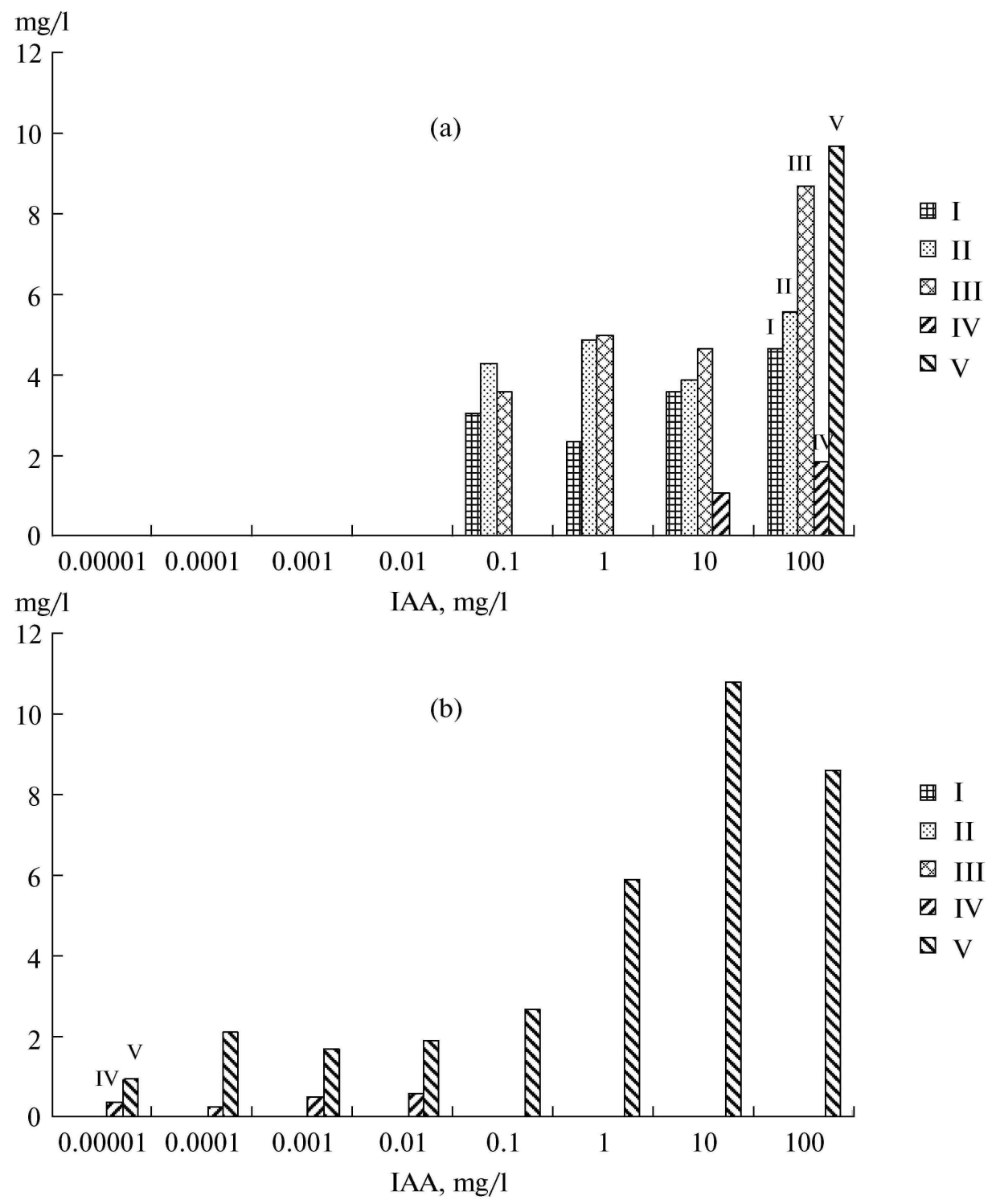

Figure 4. Effect of exogenous indolylacetic acid additives ( $\mathrm{mg} / \mathrm{l})$ on the content (mg/l) of (a) indolylacetaldehyde, and (b) indolylpyruvic acid in the cultural fluid of Lentinus edodes F-249 of different ages (days): (I) 3, (II) 7, (III) 10, (IV) 14, and (V) 21. 
Consequently, the reasons for the lack of Trp-independency of IAA biosynthesis in bacteria generally described in the literature do not take place with respect to L. edodes. The obtained results suggest that anthranilic acid or indole is a quite effective precursor to IAA as compared to Trp. It also allows for the detection of the effect of low concentrations of exogenous indolic compounds in the studied fungal culture.

\subsection{Effect of small doses of IAA in the submerged culture of Shiitake}

The biological activity of IAA at low concentrations has not been studied, the molecular mechanism of the phytohormonal action of IAA is not finally determined, and there is no explanation to the two-phase effects of heteroauxin.

In our work, where a culture of the shiitake mushroom was used as a biological object of study, it was interesting to detect the "effect of small doses" of biologically active substances of indolic nature.

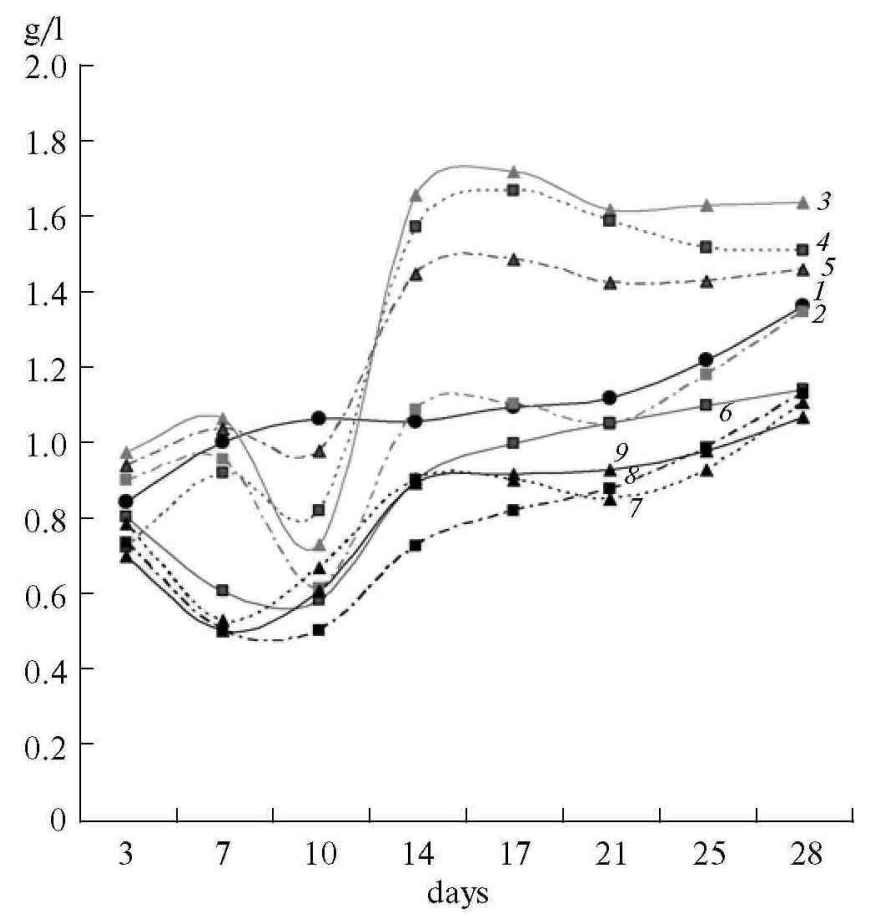

Figure 5. Mycelial biomass accumulation ( $\mathrm{g} / \mathrm{l})$ by submerged cultures of Lentinus edodes F-249 of different ages (days) on media with IAA additives (g/l): (1) 0 , (2) $10^{-8}$, (3) $10^{-7}$, (4) $10^{-6},(5) 10^{-5}$, (6) $10^{-4}$, (7) $10^{-3}$, (8) $10^{-2}$, and (9) $10^{-1}$

Under the influence of exogenous IAA, we observed growth stimulation in the submerged mycelium within a certain concentration range of auxin. It can be noted that, virtually for the entire range of values on the $x$ axis, the set of curves describing the growth of the fungus 
(dependence of the accumulation of dry biomass on the duration of cultivation) (Fig. 5) is divided into two groups of curves with the boundary curve corresponding to an IAA concentration of $10^{-4} \mathrm{~g} / \mathrm{l}$.

Above the latter, there is the area of growth activated by auxin $\left(10^{-8}-10^{-5} \mathrm{~g} / \mathrm{l}\right.$ of IAA). Below is a region with dependences with opposite properties, where the level of IAA additives is $10^{-1}-10^{-3} \mathrm{~g} / \mathrm{l}$. During the cultivation periods of 3-7 and $12-28$ days (i.e., at all studied ages of the culture, except for the interval of 8-11 days), the optimal concentration of IAA was $10^{-7} \mathrm{~g} / \mathrm{l}$.

During studying the effect of the additives of exogenous IAA on its content in the culture liquid of L. edodes, the concentration of auxin $\left(10^{-4} \mathrm{~g} / \mathrm{l}\right)$ was a turning point in the sense that the level of IAA in the medium was higher than originally (Fig. 3a). The concentration of IAA additives of $10^{-7} \mathrm{~g} / 1\left(0.57 \cdot 10^{-9} \mathrm{M}\right)$ was notable for the fact that it induced a 4100 -fold increase in the level of phytohormone.

When adding IAA, very significant stimulation of IAAld biosynthesis during the cultivation of the shiitake mushroom was observed (up to $9.7 \mathrm{mg} / \mathrm{l}$ ) on the 21st day (Fig. 4a). However, this only occurred up to $10^{-4} \mathrm{~g} / \mathrm{l}$ of IAA, below which the effect was absent.

Indole-3-pyruvic acid accumulated in the medium after 21 days of cultivation of the fungus at all concentrations of auxin (Fig. 4b), but it was only below an exogenous IAA concentration of $10^{-4} \mathrm{~g} / \mathrm{l}$ that we managed to find significant quantities of IPyA as soon as on the 14th day $(0.56 \mathrm{mg} / \mathrm{l})$.

Synthesis of extracellular anthranilic acid by submerged cultures of L.edodes F-249 of various ages on media with IAA was not observed up to a concentration of $10^{-4} \mathrm{~g} / \mathrm{l}$ (Fig. $3 \mathrm{~b}$ ), below which the concentration of anthranilate was almost $0.4 \mathrm{mg} / \mathrm{l}$ as early as the tenth day of cultivation. The maximum output of anthranilate $(1.5 \mathrm{mg} / \mathrm{l})$ was detected for the experimental variant with an inducing concentration of IAA of $10^{-7} \mathrm{~g} / 1\left(0.57 \cdot 10^{-9} \mathrm{M}\right)$.

In these omnidirectional effects of exogenous IAA, the abovementioned effect of small doses of phytohormone had a further illustration. Indeed, just as in [1], a type of a point of sign change of the biological effect of IAA was the concentration of $5.7 \bullet 10^{-7} \mathrm{M}\left(1.0 \bullet 10^{-4} \mathrm{~g} / \mathrm{l}\right)$. In addition, when IAA was used at a concentration of $10^{-9} \mathrm{M}\left(1.75 \bullet 10^{-7} \mathrm{~g} / \mathrm{l}\right)$, which, according to the just aforementioned work, promotes a more effective interaction between auxin and the membrane or the receptor, we observed absolute maxima in the respective series of experiments (the formation of IAA and anthranilate).

\subsection{Effect of IAA and its predecessors on the pigmentation of submerged mycelia of $L$. edodes F-249}

The value of phytohormones for the morphogenesis of higher xylotrophic fungi has practically not been studied. Some evidence of the involvement of IAA phytohormone in the process of morphogenetic differentiation of mushrooms of the Lentinus genus obtained through simple experiments has existed for a long time, although they are only described in a few papers $[15,16]$. These authors suggest that phytohormones play an important role in the dif- 
ferentiation of the fungal culture and that the process of morphogenesis is closely related to the dynamics of the level of endogenous growth regulators, including IAA.

Investigation of the role of phytohormonal substances in the metabolism of L. edodes is of particular relevance at the stage prior to fruiting, which is characterized in shiitake by the development of a specialized vegetative formation - brown mycelial film (BMF), because the biochemical conditions for the genesis of such morphogenetic structures are still poorly understood.

In studying the effects of IAA and its precursors, as tryptophan, tryptamine, indolylacetamide, indolylpyruvic acid, and indole, on the growth of submerged mycelia of L. edodes F-249, in most cases, there was no change in the morphology of the culture. The only exception was the medium with $0.1 \mathrm{mg} / \mathrm{l}$ of IAAm. In this medium, there was a decrease in the time required for the appearance of a brown mycelial film. In the medium with this additive, the film appeared as soon as the 17th day, whereas in the control version with other concentrations of IAAm and in the media with the remaining indole compounds, its formation was not observed.

In a study of the intensity of the growth processes of L. edodes in the presence of indolylacetamide, precisely the level of $0.1 \mathrm{mg} / \mathrm{l}$ of IAAm in the original culture medium corresponded to the largest positive effect in regard to the accumulation of mycelial biomass (Fig. 6). At the mentioned optimal concentration of IAAm, in turn, the increase in biomass was the greatest precisely on the 17 th day $(24 \%)$.

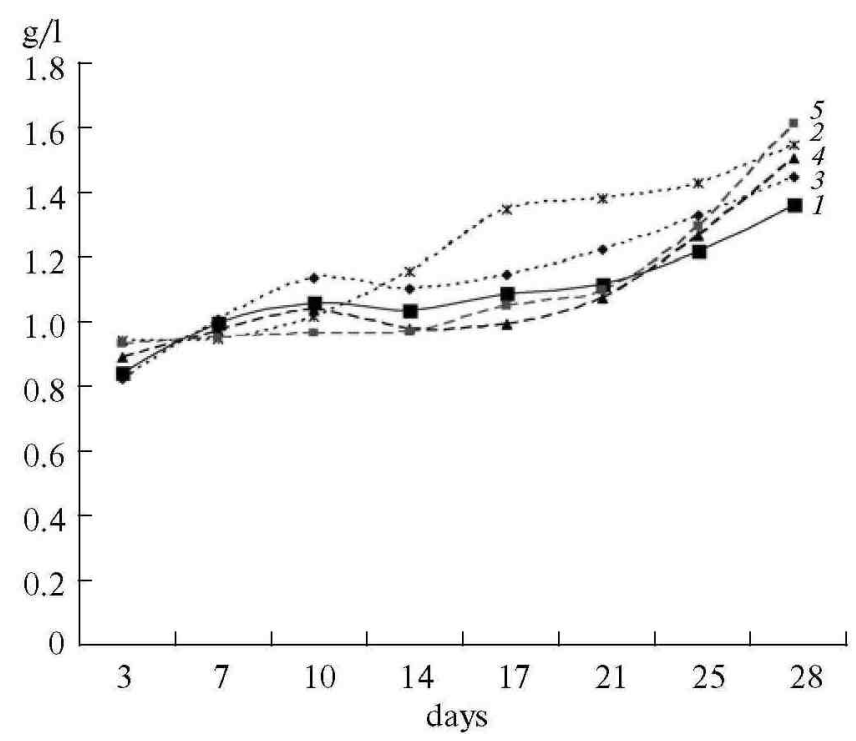

Figure 6. Mycelial biomass accumulation ( $\mathrm{g} / \mathrm{l}$ ) by submerged cultures of Lentinus edodes F-249 of different ages (days) on media with indolylacetamide additives (mg/l): (1) 0, (2) 0.1, (3) 1, (4) 10, and (5) 100. 
In the studied samples of culture fluid, 5-hydroxy-IAA, an oxidized form of IAA, was present in the control and in the media with TAM and IAAm at all ages of the mycelium, and its concentration in this case ranged from 0.78 to $2.63 \mathrm{mg} / \mathrm{l}$ (Fig. 1b). The only exception was the medium with $0.1 \mathrm{mg} / \mathrm{l}$ of IAAm, where on the 14th-21st days the level of 5-hydroxy-IAA increased dramatically. As was mentioned above, this version of the experiment differed from the rest by an early appearance of a brown mycelial film. On the 14th day, there was observed a significant pigmentation of the mycelium, and the film had completely formed by 17th day. We can assume that 5-hydroxy-IAA is involved in the formation of the BMF.

The existence of extracellular hydroxylating enzymes in mushrooms may be of ecological and environmental as well as biotechnological relevance. Biocatalytic oxygen transfer by isolated enzymes or whole microbial (fungal) cells is an elegant and efficient way to achieve selective hydroxylation. Selective hydroxylation of aromatic compounds is among the most challenging chemical reactions in synthetic chemistry and has gained steadily increasing attention during recent years, particularly because of the use of hydroxylated aromatics as precursors for pharmaceuticals [17].

The accumulation of a large biomass of mycelium is one of the factors necessarily required to make a transition to the morphogenetic stage prior to fruiting (BMF in the shiitake fungus), but not a sufficient condition for the development of a BMF. In the formation of mycelium pigmentation, oxidized indole derivatives should be also involved, the participation of which in the conversion of 5,6-dihydroxyindole-indole $\rightarrow$ 5,6-quinone $\rightarrow$ melanochrome $\rightarrow$ melanin in the case of catalysis by fungal tyrosinases leading to the formation of melanin pigments of fungi has been known for a long time [18].

Thus, during the submerged cultivation of the xylotrophic basidiomycete L. edodes, a group of metabolites of indolic nature was revealed, the composition and quantitative ratio of which indicated the coexistence of two alternative routes of IAA biosynthesis in L. edodes. These routes are Trp-dependent (mainly via tryptamine) and Trp-independent, the latter being not only implemented in the presence of exogenous indole within the concentration range of $1 \bullet 10^{-3}-1 \bullet 10^{-4} \mathrm{~g} / \mathrm{l}$, but also at inducing the biosynthesis of IAA by its exogenous microadditives. The involvement of indole-3-acetamide and 5-hydroxy-IAA in the morphogenetic processes of the shiitake culture has been revealed. There was established the interrelationship of the level of IAAm and 5-hydroxy-IAA with the formation of a brown mycelial film in the submerged culture and with the processes of growth and development of fungal mycelia during submerged cultivation.

Despite several biocatalytic processes which have successfully put on the market, much research remains to be done before enzymes can be used routinely throughout the chemical industry $[19,20]$. Enzymes as catalysts in chemical syntheses make chemical reactions possible under mild, environmentally friendly conditions in aqueous reaction mixtures and mostly, enzymes show specificity and selectivity that cannot be achieved by traditional chemical methods [21]. Enzymes implemented in the indolic compounds' biosynthesis not only in plants and bacteria, but also in mushrooms, are capable of catalyzing regio-and stereoselective transformations leading to products that are useful as fine chemicals or pharmaceuticals. 


\section{Conclusions}

An enormous body of knowledge demonstrates that auxin indolyl-3-acetic acid can be synthesized from tryptophan by plants and bacteria. Only few experiments are done with fungi, therewith the published works on phytohormonic potentialities of xylotrophic mushrooms are virtually absent.

The characterization of the group of extracellular indolic compounds of basidiomycete Lentinus edodes in relation to the submerged cultivation conditions has been presented. The L. edodes F-249 culture has been stated to synthesize the indolic-nature compounds when being grown in submerged culture. The group of extracellular indolic compounds of shiitake includes, in different proportions, the following components: L-tryptophan, $\beta$-indolyl-3-acetic acid, $\beta$-indolyl-3-acetaldehyde, $\beta$-indolyl-3-acetamide, indolyl-3-pyruvic acid, tryptamine, 5hydroxy- $\beta$-indolyl-3-acetic acid.

The time-course production of indolic derivatives in culture liquid of shiitake has been studied comparatively to reveal correlations with the growth rates. It has been established that shiitake is capable of producing indolylacetic acid rather actively in the culture-age dependent manner. Maximal indolylacetic acid content we marked in experiments was $10.6 \mathrm{mg} / \mathrm{l}$ provided that the initial tryptamine level in the medium was $1 \mathrm{mg} / \mathrm{l}$. The highest levels of tryptamine $(22.8 \mathrm{mg} / \mathrm{l})$, indolylpyruvic acid (13.9 mg/l), indolylacetaldehyde (27.9 mg/l), 5hydroxy-indolylacetic acid $(9.2 \mathrm{mg} / \mathrm{l})$ have been observed at different additives of the indole-group substances, and varied with the fungal culture age. The indolylacetamide level changed only slightly as compared to reference probe. Starting from the synthetic tryptophan-free medium, the concentrations of extracellular tryptophan became from $13.8 \mathrm{mg} / \mathrm{l}$ (7th day) to $23.9 \mathrm{mg} / \mathrm{l}$ (21st day).

On the basis of literature data on physiologically active concentration values for auxins in plants, we use the concentration range of $10^{-1}-10^{-8} \mathrm{~g} / \mathrm{l}$ of additives to explore the effects of indolylacetic acid and its precursors upon the shiitake submerged culture. The positive influence of exogenic auxin within its concentration range of $2 \bullet 10^{-7}$ to $2 \bullet 10^{-4} \mathrm{~g} / \mathrm{l}$ upon the $L$. edodes biomass accumulation has been found, a minimal growth-inhibiting phytohormone concentration being about $5 \bullet 10^{-4} \mathrm{~g} / 1$ on mineral medium. When inducing the indolylacetic acid biosynthesis by its exogenous micro-additives $\left(1 \bullet 10^{-5}\right.$ to $\left.1 \bullet 10^{-8} \mathrm{~g} / \mathrm{l}\right)$, the increase in phytohormone level in the medium (up to 4000 -fold) accompanied by the anthranilic acid appearance (up to $1.5 \mathrm{mg} / \mathrm{l}$ ) have been revealed under these experimental conditions exclusively.

The studies of biosynthetic routes for indolylacetic acid realized by the mushroom culture under question have been attempted in order to conclude whether the above route is tryptophan-dependent, or tryptophan-independent pathway becomes also involved when the mushroom grows in the presence of exogenous synthetic analogs of the auxin precursors. The experimental evidences in favor of co-existence of two alternative pathways for indolylacetic acid production by L. edodes have been obtained. Those routes are: tryptophan-dependent (mainly via tryptamine) and tryptophan-independent, the latter being realized in 
the presence of exogenous indole within the concentration range $1 \bullet 10^{-3}-1 \bullet 10^{-4} \mathrm{~g} / \mathrm{l}$ or the auxin micro-additives.

The induction of generative developmental stage by indolic derivative has been revealed for shiitake. It has been stated that among the compounds - indolylacetic acid precursors, solely indolylacetamide at a concentration of about $10^{-4} \mathrm{~g} / \mathrm{l}$ in L. edodes culture liquid exerts the explicitly marked stimulating effect on the occurrence of morphological structure - brown mycelial film.

More thorough investigations and specific search for the extracellular indolic compounds among the huge number of basidiomycetous fungi colonizing litter or lignicelluloses will surely result in the discovery of further fungal enzymes, as well as may help to understand better the biological formation of the mushroom growth promoting substances and polycyclic aromatic compounds in terrestrial ecosystems.

\section{Acknowledgements}

This work was supported in part by the "OOO Sibirskoye zdorov'e" Russian Foundation, grant of the series "Useful properties of plants and minerals for the medicinal cosmetics and natural prophylactic preparations production - 2012".

\section{Author details}

Olga M. Tsivileva*, Ekaterina A. Loshchinina and Valentina E. Nikitina

*Address all correspondence to: tsivileva@ibppm.sgu.ru

Laboratory of Microbiology, Institute of Biochemistry and Physiology of Plants and Microorganisms, RAS, Saratov, Russia

\section{References}

[1] Rogacheva, S.M., Rol' vodnoi komponenty i polisakharidov kletochnoi poverkhnosti v protsessakh kommunikatsii zhivykh sistem: analiz molekulyarnykh modelei (The Role of Aqueous Components and Cell Surface Polysaccharides in the Communication Processes of Living Systems: Analysis of Molecular Models), Voronezh: Izd. Voronezh. GOs. Univ., 2008. http://vak.ed.gov.ru/ru/dissertation/index.php?id54=1099

[2] Burlakova, E.B., Konradov, A.A., and Mal'tseva, E.L. Extremely small actions of chemical compounds and physical factors on biological systems. Biophysics, 2004, vol. 49 , no. 3, pp. 522-534. 
[3] Baca, B.E. and Elmerich, C. Associative and Endophytic Nitrogen-Fixing Bacteria, and Cyanobacterial Associations, in Series: Nitrogen Fixation: Origins, Applications, and Research Progress, V. 5, Elmerich, C. and Newton, W.E., Eds., Dordrecht, The Netherlands: Springer, 2007, chapter 6, pp. 113-143.

[4] Eckardt, N.A. New Insights into Auxin Biosynthesis. Plant Cell, 2001, vol. 13, no. 1, pp. 1-3.

[5] Reineke, G., Heinze, B., Schirawski, J., Buettner, H., Kahmann, R., and Basse, C.W. Indole-3-acetic acid (IAA) biosynthesis in the smut fungus Ustilago maydis and its relevance for increased IAA levels in infected tissue and host tumour formation. Mol. Plant Pathol., 2008, vol. 9, no. 3, pp. 339-355.

[6] Prinsen, E., Costacurta, A., Michiels, K., Vanderleyden, J., and Van Onckelen, H. Azospirillum brasilense indole-3-acetic acid biosynthesis: evidence for a non-tryptophan dependent pathway. Mol. Plant-Microbe Interact., 1993, vol. 6, no. 5, pp. 609-615.

[7] Bar, T. and Okon, Y., in Azospirillum VI and Related Microorganisms: Genetics, Physiology, Ecology, Fendrik, I., Gallo, M. D., Vanderleyden, J., and Zamaroczy, M., Eds., Berlin: Springer, 1995, pp. 347-359.

[8] Costacurta, A., Keijers, V., and Vanderleyden, J. Molecular cloning and sequence analysis of an Azospirillum brasilense indole-3-pyruvate decarboxylase gene. Mol. Gen. Genet., 1994, vol. 243, no. 4, pp. 463-472.

[9] Loshchinina, E.A., Tsivileva, O.M., Nikitina, V.E., and Makarov, O.E., Vavilovskie Chteniya-2008: Materialy mezhd. nauchno-prakt. konf (Vavilov Reading - 2008: Proceedings of the Int. Scientific and Practical. Conf.), Saratov: ITs Nauka, 2008, pp. 30-32.

[10] Lebuhn, M., Heulin, T., and Hartmann, A. Production of auxin and other indolic and phenolic compounds by Paenobacillus polymyxa strains isolated from different proximity to plant roots. FEMS Microbiol. Ecol., 1997, vol. 22, no. 4, pp. 325-334.

[11] Bar, T. and Okon, Y. Induction of indole-3-acetic acid synthesis and possible toxicity of tryptophan in Azospirillum brasilense Sp 7. Symbiosis, 1992, vol. 13, nos. 1-3, pp. 191-198.

[12] Lebuhn, M. and Hartmann, A. Production of auxin and ltryptophan related indolic and phenolic compounds by Azospirillum brasilense and Azospirillum lipoferum. in Improving Plant Productivity with Rhizosphere Bacteria, Ryder, M.H., Stephens, P.M., and Bowen, G.D., Eds., Australia: CSIRO, 1994, pp. 145-147.

[13] Zakharova, E.A., Shcherbakov, A.A., Brudnik, V.V., Skripko, N.G., Bulkhin, N.Sh., and Ignatov, V.V. Biosynthesis of indole-3-acetic acid in Azospirillum brasilense. Insights from quantum chemistry. Eur. J. Biochem., 1999, vol. 259, no. 3, pp. 572-576.

[14] Dewick, P.M. The biosynthesis of shikimate metabolites. Nat. Prod. Rep., 1998, vol. 15, no. 1, pp. 17-58. 
[15] Rypacek, V. and Sladky, Z. The character of endogenous growth regulators in the course of development in the fungus Lentinus tigrinus. Mycopathol. Mycol. Applic., 1972, vol. 46, no. 1, pp. 65-72.

[16] Rypacek, V. and Sladky, Z. Relation between the level of endogenous growth regulators and the differentiation of the fungus Lentinus tigrinus studied in a synthetic medium. Biologia Plantarum (Praha), 1973, vol. 15, no. 1, pp. 20-26.

[17] Ullrich, R. and Hofrichter, M. Enzymatic hydroxylation of aromatic compounds. Cell. Mol. Life Sci., 2007, vol. 64, no. 3., pp. 271-293.

[18] Choi, S.W. and Sapers, G.M. Purpling Reaction of Sinapic Acid Model Systems Containing L-DOPA and Mushroom Tyrosinase. J. Agric. Food Chem., 1994, vol. 42, no. 5, pp. 1183-1189.

[19] Schoemaker, H.E., Mink, D., and Wubbolts, M.G. Dispelling the myths - biocatalysis in industrial synthesis. Science, 2003, vol. 299, pp. 1694-1697.

[20] Poliakoff, M, Fitzpatrick, J.M., Farren, T.R., and Anastas, P.T. Green chemistry: science and politics of change. Science, 2002, vol. 297, pp. 807-810.

[21] Hofrichter, M., and Ullrich, R. Heme-thiolate haloperoxidases: versatile biocatalysts with biotechnological and environmental significance. Appl. Microbiol. Biotechnol., 2006, vol. 71, no. 3, pp. 276-288. 